\title{
Pegylated and nanoparticle-conjugated sulfonium salt photo triggers necrotic cell death
}

\author{
This article was published in the following Dove Press journal: \\ International Journal of Nanomedicine \\ 18 November 2016 \\ Number of times this article has been viewed
}

\author{
Alaa A Fadhel' \\ Xiling Yue' \\ Ebrahim H Ghazvini Zadeh' \\ Mykhailo V Bondar ${ }^{2}$ \\ Kevin D Belfield ${ }^{3}$ \\ 'Department of Chemistry, University \\ of Central Florida, Orlando, FL, USA; \\ ${ }^{2}$ Institute of Physics NASU, Kiev, \\ Ukraine; ${ }^{3}$ Department of Chemistry \\ and Environmental Science, College of \\ Science and Liberal Arts, New Jersey \\ Institute of Technology, University \\ Heights, Newark, NJ, USA
}

\begin{abstract}
Photodynamic therapy (PDT) processes involving the production of singlet oxygen face the issue of oxygen concentration dependency. Despite high oxygen delivery, a variety of properties related to metabolism and vascular morphology in cancer cells result in hypoxic environments, resulting in limited effectiveness of such therapies. An alternative oxygen-independent agent whose cell cytotoxicity can be remotely controlled by light may allow access to treatment of hypoxic tumors. Toward that end, we developed and tested both polyethylene glycol (PEG)-functionalized and hydrophilic silica nanoparticle (SiNP)-enriched photoacid generator (PAG) as a nontraditional PDT agent to effectively induce necrotic cell death in HCT-116 cells. Already known for applications in lithography and cationic polymerization, our developed oxygen-independent PDT, whether free or highly monodispersed on SiNPs, generates acid when a one-photon (1P) or two-photon (2P) excitation source is used, thus potentially permitting deep tissue treatment. Our study shows that when conjugated to SiNPs with protruding amine functionalities (SiNP-PAG9), such atypical PDT agents can be effectively delivered into HCT-116 cells and compartmentalize exclusively in lysosomes and endosomes. Loss of cell adhesion and cell swelling are detected when an excitation source is applied, suggesting that SiNP-PAG9, when excited via near-infrared 2P absorption (a subject of future investigation), can be used as a delivery system to selectively induce cell death in oxygen-deprived optically thick tissue.
\end{abstract}

Keywords: oxygen-independent photodynamic therapy, photoacid generator, silica nanoparticles, stimuli-responsive, sulfonium salt

\section{Introduction}

The clinical usefulness of light was first recognized some 3,000 years ago when it was used to treat rickets, psoriasis, and other common ailments. ${ }^{1}$ More recently, the term photodynamic therapy (PDT) surfaced and has been used to describe the use of light to treat a variety of diseases, including cancer. ${ }^{2-5}$ Specifically, over the last 100 years, great strides have been made in developing photodynamic therapies for use in the clinical setting. PDT hinges on a few key components. The first component is the photosensitizer, the photosensitive material that targets diseased tissue, akin to chemotherapeutic agents. Biocompatibility and potential for targeting are important to the optimization of this component. The second component is the irradiation of the target area with light of a specific wavelength. Excitation of the photosensitive material to a triplet state in the presence of oxygen results in triplet-triplet annihilation, producing cytotoxic reactive oxygen species (ROS) that destroy cellular components, thus triggering cell death. Therefore, the success of photodynamic therapeutics depends largely on the photosensitizer concentration throughout the tissue, adequate intracellular 
oxygen concentration, and proper source of excitation. The discovery of this process was extremely promising in providing a more targeted, regulated method of cancer treatment. However, one of the main drawbacks of this method of PDT is that it is oxygen dependent. Although cancer cells have abnormally high vascularization, intracellular oxygen concentration is low due to poor morphology of the vessels and abnormally high metabolic and oxygen consumption rates, which are characteristics of cancerous tissues. ${ }^{2} \mathrm{Without}$ oxygen available to interact with the photosensitizer, ROS cannot be formed and the therapeutic method becomes relatively ineffective or far less effective than intended.

\section{Approach}

In addressing the aforementioned issue, we recently took an alternative approach in attempting to trigger cell death by employing a photoresponsive agent with low dark cytotoxicity. Rather than producing ROS as the main "damaging" component, we used a non-toxic sulfoniumbased photoacid generator (PAG) to cause intracellular $\mathrm{pH}$ imbalance. ${ }^{6}$ Upon proper light excitation, the PAG lowers the $\mathrm{pH}$ in the moderately $\mathrm{pH}$-sensitive intracellular environment; the acidic environment thus created leads to the malfunction and destruction of many cellular components, likely including key enzymes. This process is considered an oxygen-independent PDT (OI-PDT) and, essentially, relies on two main components, the sulfonium salt distribution in the affected area and a proper source of excitation, rather than the three components mentioned earlier. In essence, reducing the number of variables in the therapeutic process development improves the chances of success in material design and subsequent treatment.

Given the hydrophobic nature of PAGs prepared in our preliminary study, ${ }^{6}$ we opted to introduce a polyethylene glycol (PEG) unit that can confer hydrophilicity to the PDT agent while maintaining its photo-induced cytotoxicity. Along the same line, we prepared highly monodispersed silica nanoparticles (SiNPs) functionalized with amines that served as handles to covalently bond PAG molecules via an amide linkage. The incorporation of the PAG into a nanomaterial delivery formulation is also intended to improve delivery properties of the proposed PDT agent as nanomaterials have emerged as a promising solution to the issues of cellular specificity, time-controlled delivery, and aqueous solubility. ${ }^{7,8}$ Herein, we report the design, synthesis (Scheme 1 and Supplementary material), and investigation of a new PAG and its incorporation on SiNPs (SiNP-PAG9), which offers better solubility in aqueous media, greater efficacy than water-soluble PEGylated PAG (PEG-PAG9), and low dark cytotoxicity.

PEG-PAG9 and SiNP-PAG9 were designed having similar $\mathrm{A}-\pi-\mathrm{A}^{\prime}$ core structure to the one reported in our initial work, where the two acceptor units (A), $-\mathrm{NO}_{2}$ and $-\mathrm{SPh}_{2}{ }^{+}$, flank a fluorenyl-stilbene $\pi$-spacer. Using the $C(9)$ of fluorene 1 to attach a handle for further functionalization, a propanoic acid unit was added as depicted in Scheme 1. Initially, treatment of 1 with one equivalent of $n$-BuLi at $-78^{\circ} \mathrm{C}$ generated a carbanion, stabilized by resonance through the fluorene $\pi$-system; this was followed by careful addition of ethyl bromide to afford the monoalkylated intermediate 2 . Bi-substituted fluorene $\mathbf{3}$ was obtained through a Triton B-assisted Michael addition of $\mathbf{2}$ with acrylonitrile in satisfactory yield (77\%).

\section{Materials and methods}

Although later stages of the synthesis that use aqueous acids have resulted in the hydrolysis of the nitrile moiety, such acidic hydrolysis was incomplete and often resulted in low yields and multiple side products. Therefore, we opted to hydrolyze the nitrile group using aqueous $\mathrm{NaOH}$ to afford the carboxylic acid derivative $\mathbf{4}$ in good yield. Subsequent intermediates 5-9 were prepared following our reported procedure to afford PAG 9 in $\sim 61 \%$ yield over four steps. This compound was conjugated to an amine-terminated PEG moiety by preparing the benzotriazole intermediate in situ, followed by the addition of the amine-terminated PEG to afford PEG-PAG9 in 77\% yield. On the other hand, SiNPs having amine appendages $\operatorname{SiNP}\left(\mathrm{NH}_{2}\right)_{\mathrm{n}}$ (preparation detailed in the Supplementary material) were treated with 1-ethyl3-(3-dimethylaminopropyl)carbodiimide condensation agent, followed by the addition of an excess of PAG 9; the good solubility of PAG 9 in water allowed the dialysis of the resulting SiNP-PAG9 to separate any unreacted PAGs. Surface modification of $\operatorname{SiNP}\left(\mathrm{NH}_{2}\right)_{\mathrm{n}}$ by addition of PAG 9 molecules was first assessed using a Zetasizer Nano system (Malvern Instruments, Malvern, UK), where such addition resulted in particle size increase from an average of $4 \mathrm{~nm}$ to $9 \mathrm{~nm}$ (Supplementary material). In addition, the nuclear magnetic resonance (NMR) spectrum of SiNP-PAG9 in $\mathrm{D}_{2} \mathrm{O}$ (Supplementary material) showed the presence of peaks in the aromatic region that corresponded to PAG 9, hence verifying the successful functionalizing of the SiNPs with the PDT (PAG) molecules.

Next, the photophysical properties of PEG-PAG9 and SiNP-PAG9 were examined to ensure that the magnitude of photoacid generation is preserved after modification. 


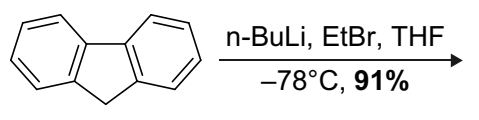

1

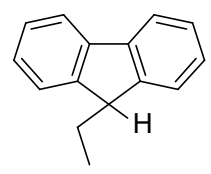

2

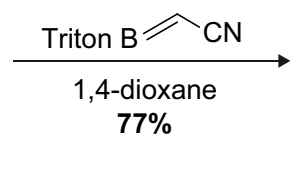

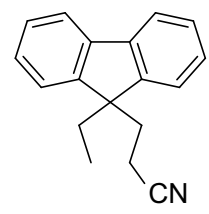

3

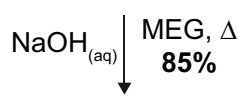

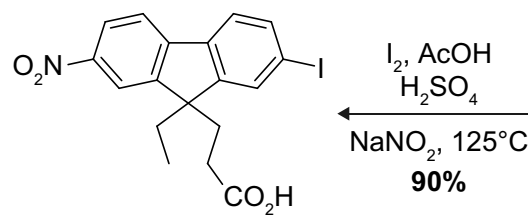

6

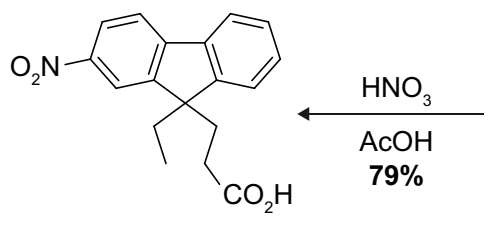

5<smiles>CCC1(CCC(=O)O)c2ccccc2-c2ccccc21</smiles>

4
8

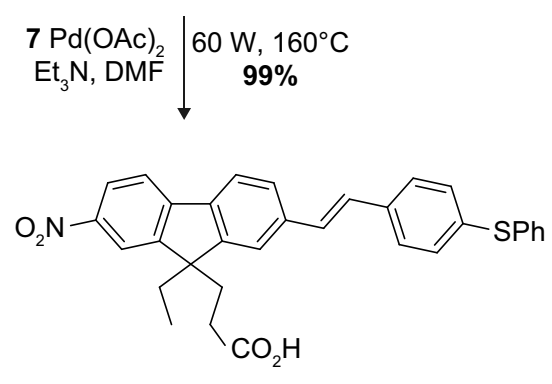

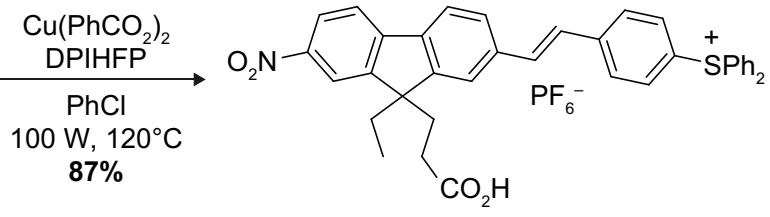

9

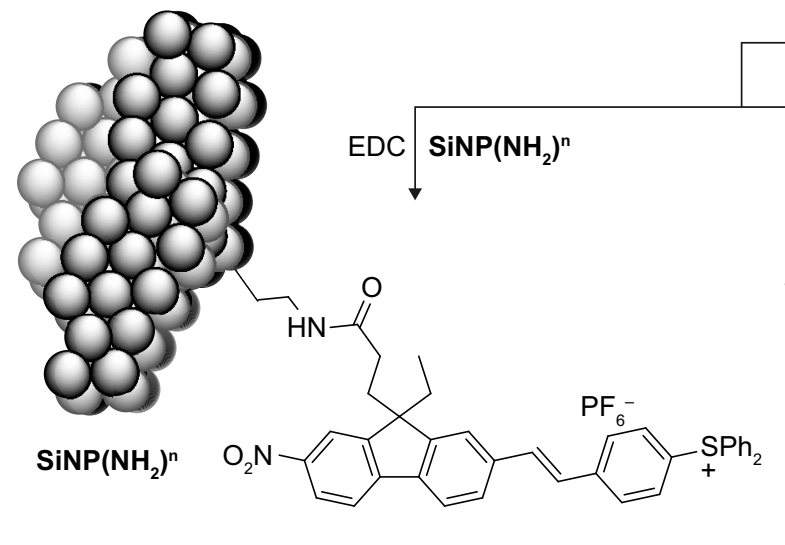

SiNP-PAG9

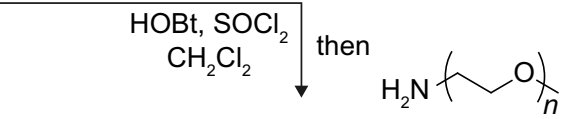

${ }^{\text {tro }} \underbrace{}_{\mathrm{HN}}$

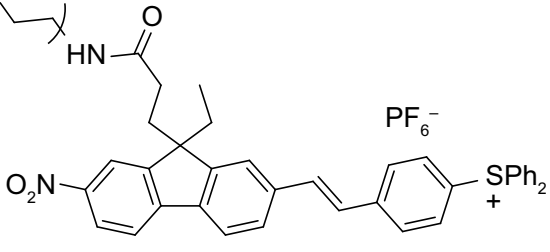

PEG-PAG9

Scheme I Synthetic route for the preparation of PEG-PAG9 and SiNP-PAG9.

Note: "7" is phenyl 4-vinylphenyl sulfide.

Abbreviations: PEG, polyethylene glycol; PAG, photoacid generator; SiNP, silica nanoparticle; THF, tetrahydrofuran; MEG, monoethylene glycol; DMF, dimethylformamide; EDC, I-ethyl-3-(3-dimethylaminopropyl)carbodiimide; DPIHFP, diphenyliodonium hexafluorphosphate.

Initially, the absorption profiles of PEG-PAG9 and SiNPPAG9 were obtained in water, where both show a similar absorption band to previously reported PL- $127^{\circledR}$-encapsulated PAG, with an absorption $\lambda_{\max }$ of $384 \mathrm{~nm}$. More importantly, the photoacid generation quantum yield $\left(\Phi_{\mathrm{H}^{+}}\right)$of PEG-PAG9 was maintained at $\sim 0.4$ (Supplementary material), suggesting that the aqueous environment has little effect on the relevant photophysical properties of PEG-PAG9. Furthermore, the $\mathrm{pH}$ drop inside cell lysosomes was estimated by recording the changes in the absorption intensity of Rhodamine B (RhB) base. Conceptually, it is safe to assume that the number of protons generated by PEG-PAG9 upon exposure is the same as the number of RhB base molecules converted to $\mathrm{RhB}+$, which is visualized as an increase in the absorption peak at ca. $555 \mathrm{~nm}$ (Figure 1A). Extrapolation of the calibration curve (Figure 1B) suggests that $50 \mu \mathrm{M}$ of PEG-PAG9 would result in an increase of $\left[\mathrm{H}^{+}\right]_{\text {lysosomal }}$ by $9.1 \times 10^{-6} \mathrm{M}$ after a radiation dose of $10 \mathrm{~min}$; consequently, the lysosomal $\mathrm{pH}$ would be 
A

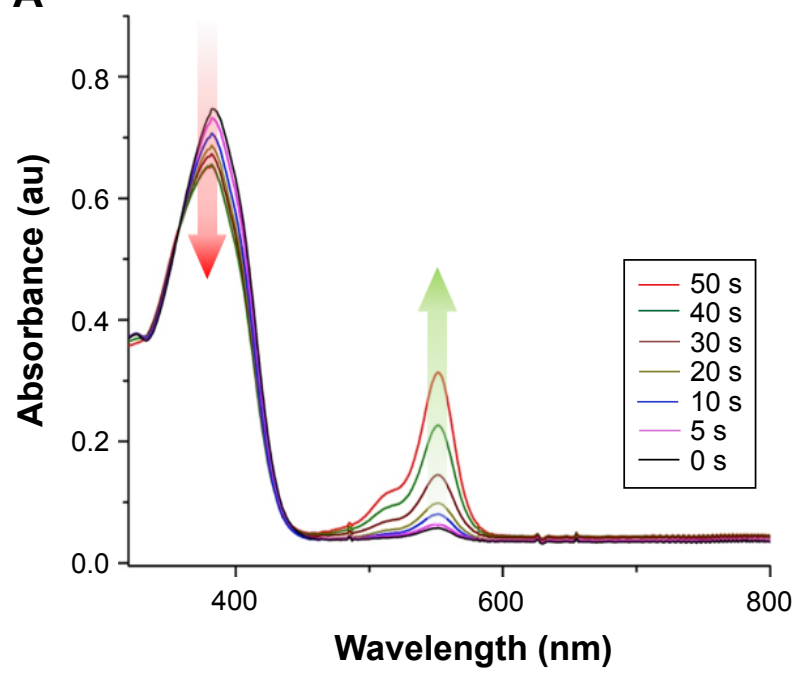

B

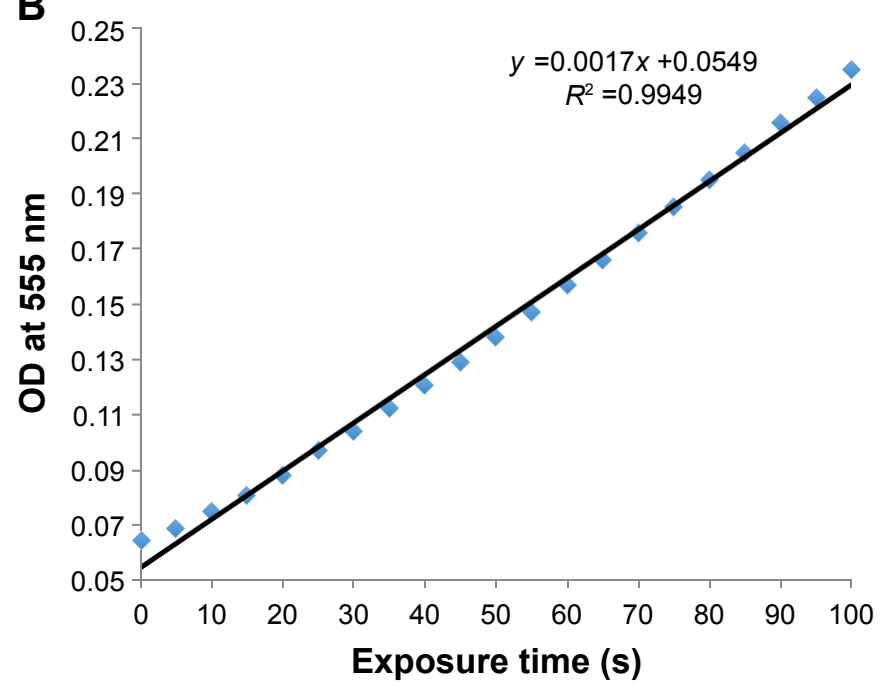

Figure I (A) Absorption spectra of PEG-PAG9 $\left(10^{-4} \mathrm{M}\right)$ and $\mathrm{RhB}$ base $\left(10^{-5} \mathrm{M}\right)$ (indicator) in $\mathrm{CH}_{2} \mathrm{Cl}_{2}$ irradiated at $366 \mathrm{~nm}$ at different time intervals. (B) Dose-dependent calibration curve using $5.4 \mathrm{~mW} / \mathrm{cm}^{2}$ irradiation.

Abbreviations: PEG, polyethylene glycol; PAG, photoacid generator; RhB, Rhodamine B; au, arbitrary units; OD, optical density.

reduced by at least $0.3 \mathrm{pH}$ units to $\sim \leq 4.4$ (Supplementary material). ${ }^{9}$

\section{Results}

In order to evaluate the intrinsic toxicity of PEG-PAG9 and SiNP-PAG9, cell viability assays with CellTiter $96{ }^{\circledR}$ AQueos One Solution Reagent (Promega Corporation, Fitchburg, WI, USA) were performed in the dark (dark viability) to prevent the production of acid and determine suitable concentration for effective PDT (Supplementary material). In this regard, HCT-116 cells (human colorectal carcinoma) were incubated with various concentrations of PEG-PAG9 and SiNP-PAG9 for $24 \mathrm{~h}$ at $37^{\circ} \mathrm{C}$. The results showed that PEG-PAG9 has very low dark cytotoxicity, even at $100 \mu \mathrm{M}$ dose concentration (Figure 2A). On the other hand, cell viability decreased from $99 \%$ to $75 \%$ when SiNP-PAG9 loading was doubled from $10 \mu \mathrm{M}$ to $20 \mu \mathrm{M}$ (Figure 2B). In order to further assess the cytotoxicity of PDT agents, post-exposure viability assays were performed using $50 \mu \mathrm{M}$ and $10 \mu \mathrm{M}$ of PEG-PAG9 and SiNP-PAG9, respectively. As can be seen in Figure 3, exposure of HCT-116 cultures incubated with PEG-PAG9 prompted a drop from $97 \%$ viability to $64 \%$ viability within $10 \mathrm{~min}$. Within the same exposure timeframe, cultures incubated with SiNP-PAG9 experienced a reduction in viability from $98 \%$ to $42 \%$. Such a drastic decrease in cell viability can be the result of PAG distribution within the lysosomes
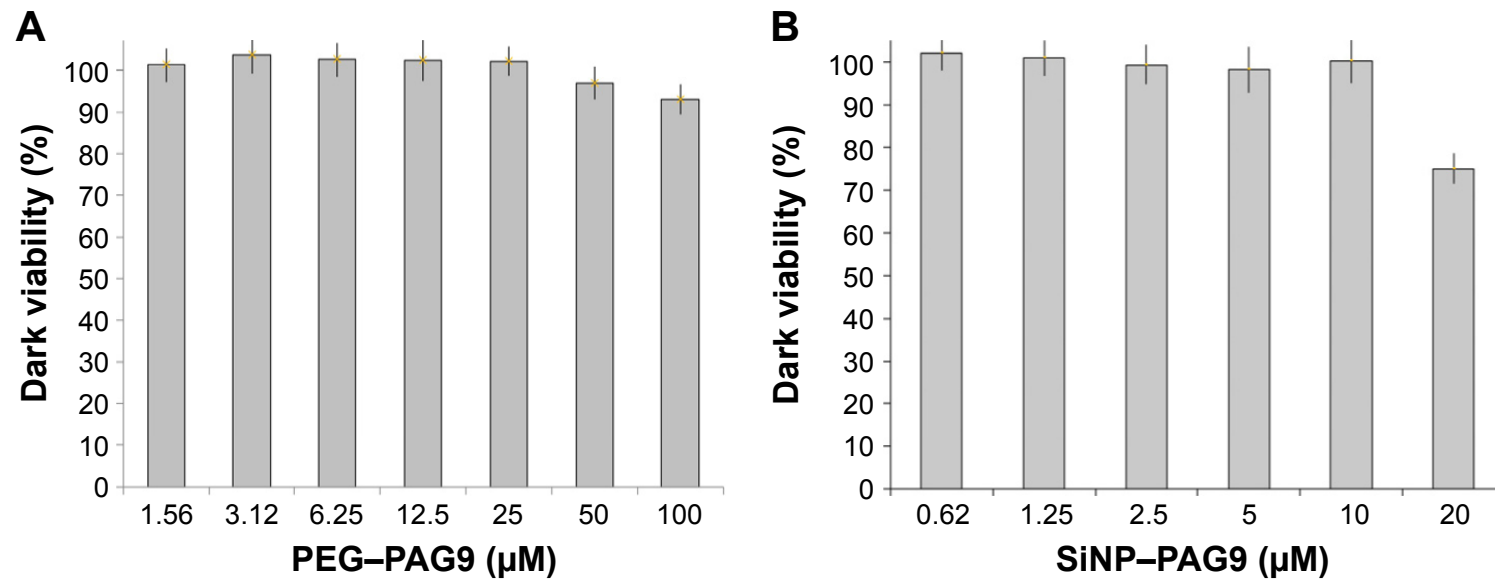

Figure 2 Dark cytotoxicity of HCT-II 6 cells incubated with (A) PEG-PAG9 and (B) SiNP-PAG9.

Abbreviations: PEG, polyethylene glycol; PAG, photoacid generator; SiNP, silica nanoparticle. 
A

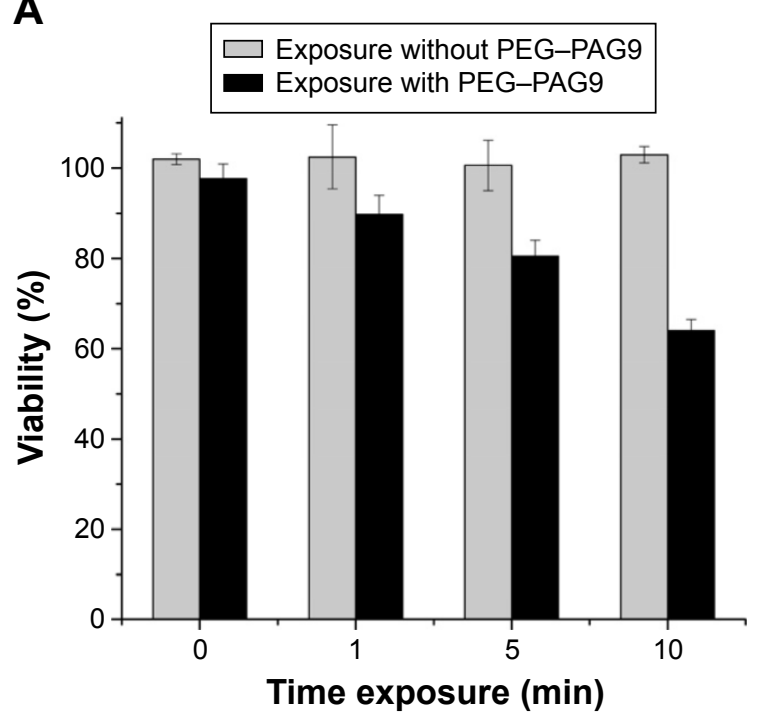

B

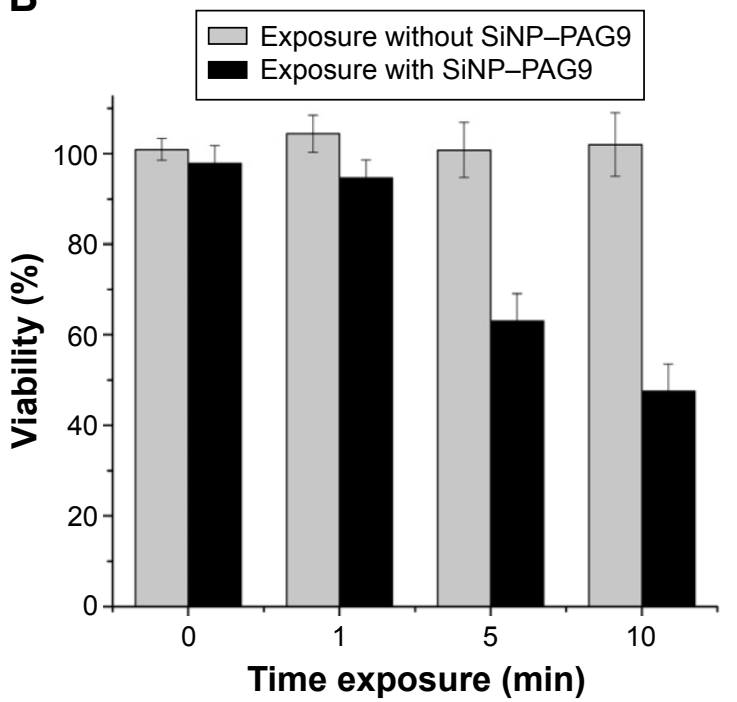

Figure 3 Post-exposure $\left(377 \mathrm{~nm}, 5.4 \mathrm{~mW} / \mathrm{cm}^{2}\right)$ viability of HCT-I I6 cells with (A) PEG-PAG9 (50 $\mu$ M) and (B) SiNP-PAG9. Abbreviations: PEG, polyethylene glycol; PAG, photoacid generator; SiNP, silica nanoparticle.

and endosomes. Mechanistically, photoexcitation of PAG 9 molecules that are densely grafted on $\operatorname{SiNP}\left(\mathrm{NH}_{2}\right)_{\mathrm{n}}$ generates a highly localized dose of protonated species, and, thus, a greater localized $\mathrm{pH}$ drop. Consequently, a localized surge in the protonated species concentration creates an acidic microenvironment that could override mechanisms by which cells tend to regulate intracellular $[\mathrm{H}]^{+}$, resulting in severe cytoplasmic acidification and inducing cell death. ${ }^{10}$ Although this report is largely qualitative, the error bars of the cell viability in Figures 2 and 3 provide some insight into the statistical significance of the results. Thus, SiNP-PAG9 exhibits high cytotoxicity at very low concentration and short exposure time.
In order to determine the localization of PEG-PAG9 in HCT 116 cells, LysoTracker Green (Thermo Fisher Scientific, Waltham, MA, USA), a commercial dye that localizes in lysosomes after cell uptake, was used along with PEG-PAG9. Fluorescence of PEG-PAG9 was collected inside cells (Figure 4A-C), showing a good uptake efficiency of PEGPAG9. Overlay image (Figure 4D) exhibited good colocalization between PEG-PAG9 and LysoTracker Green, which indicated that PEG-PAG9 mainly built up in lysosomes and endosomes. Next, we verified the reduced lysosomal $\mathrm{pH}$ by employing LysoSensor Green as a suitable intracellular $\mathrm{pH}$ sensor. ${ }^{6}$ Since the fluorescence quantum yield of LysoSensor Green increases in increasingly acidic environments, ${ }^{11}$
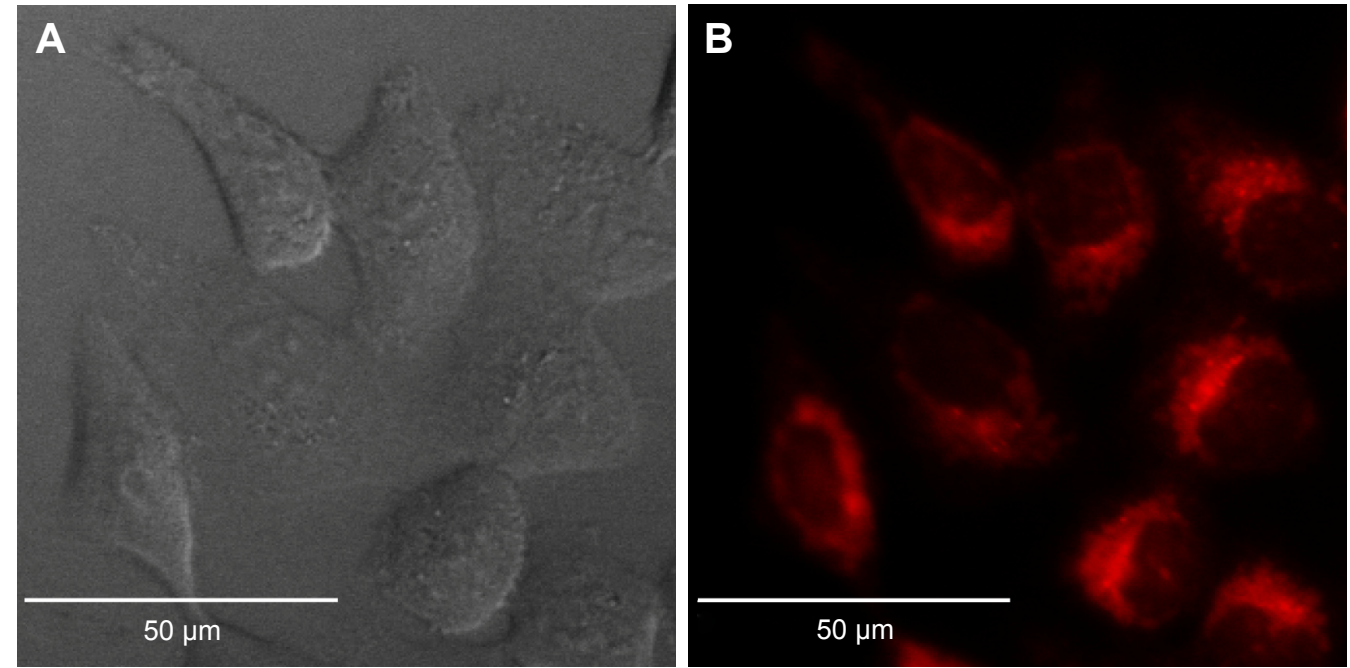

Figure 4 (Continued) 

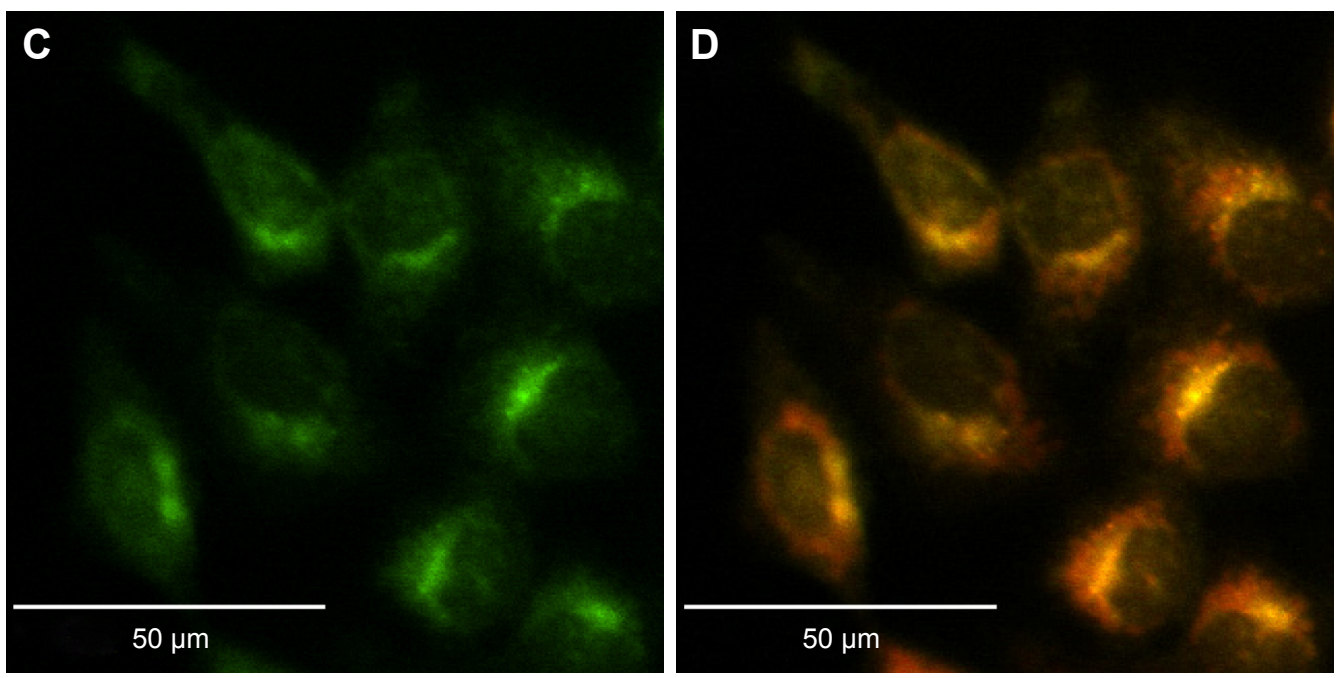

Figure 4 Lysosome colocalization experiments.

Notes: (A) DIC and confocal fluorescence micrographs of HCT-II6 cells incubated with PEG-PAG9 (B; red) and LysoTracker Green (C). Overlay image (D) shows PEG-PAG9 mainly built up in lysosomes and endosomes. Pearson's correlation coefficient $=0.92$.

Abbreviations: DIC, differential interference contrast; PEG, polyethylene glycol; PAG, photoacid generator.

fluorescence intensity as a function of exposure time was studied, which showed a drop in intralysosomal pH following irradiation in HCT-116 cells incubated with PEG-PAG9 (Figure 5 and Supplementary material).

As demonstrated in our previous study, ${ }^{6}$ time-lapsed micrographs (Figure 6) of HCT-116 cells incubated with PEG-PAG9 and SiNP-PAG9 (Figures 6 and 7, respectively) show significant cell swelling, loss of cell adhesion that is followed by a "blebbing"-like activity, which is a characteristic of necrotic cell death. Figure 8 shows a simplified Jablonski diagram summarizing the process of photoacid generation by either one-photon or two-photon (2P) excitation.

\section{Conclusion}

We demonstrated that sulfonium salts can be used to selectively induce cell death by photoexcitation. PAGs induced necrotic cell death by creating a $\mathrm{pH}$ imbalance in the HCT-116 cells via generation of photoacid within the lysosomes. OI-PDT was shown to be effective using water-soluble PAG molecules. Higher efficacy was achieved when these PAG molecules were conjugated to silica-based nanoparticles,
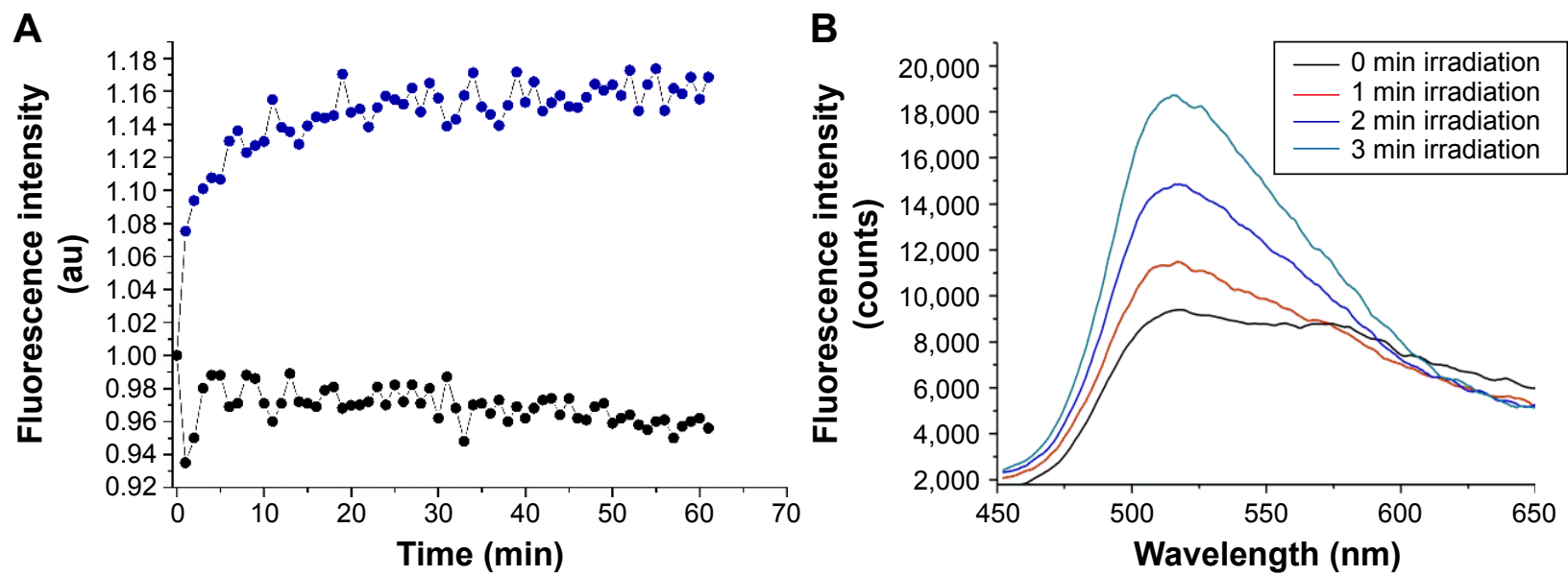

Figure 5 Demonstration of $\mathrm{pH}$ decrease and acid generation.

Notes: (A) Acidic content increasing in the lysosomes as a function of exposure time in HCT-II6 cells incubated with PEG-PAG9 (50 $\mu$ M) for $24 \mathrm{~h}$ and with I $\mu$ M of LysoSensor Green for $2 \mathrm{~h}$ after $5 \mathrm{~s}$ irradiation, $5.4 \mathrm{~mW} / \mathrm{cm}^{2}$ (blue dots). Black dots represent cells incubated only with LysoSensor Green. (B) Increase in fluorescence intensity of LysoSensor Green in the presence of PEG-PAG9 at different irradiation times.

Abbreviations: PEG, polyethylene glycol; PAG, photoacid generator. 

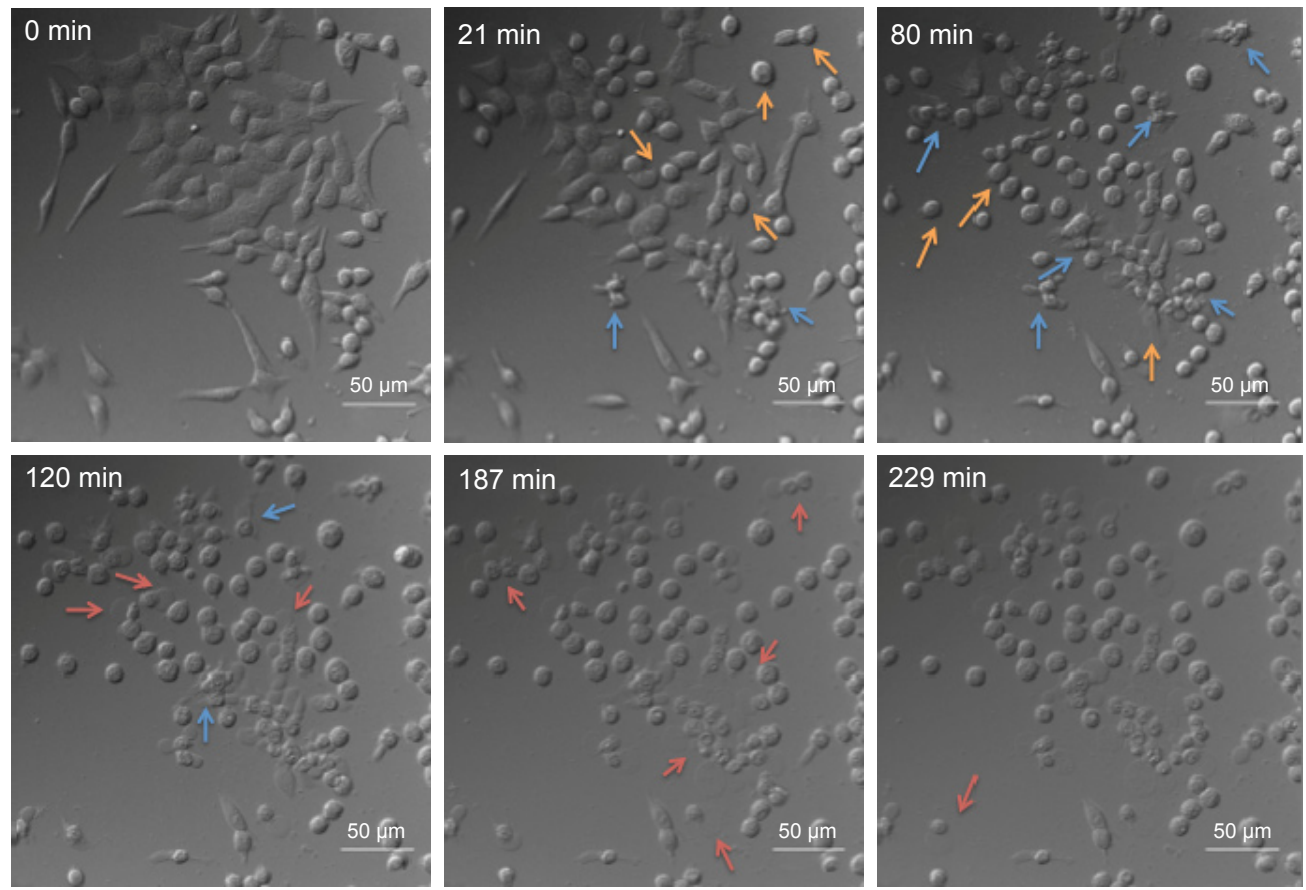

Figure 6 Time-lapse images of HCT-I I 6 cells incubated with PEG-PAG9 show the process of cell death by light irradiation $\left(5.4 \mathrm{~mW} / \mathrm{cm}^{2}\right)$.

Notes: Orange arrows show loss of cell adhesion, blue arrows show blebbing-like activity, and red arrows show cell swelling.

Abbreviations: PEG, polyethylene glycol; PAG, photoacid generator.
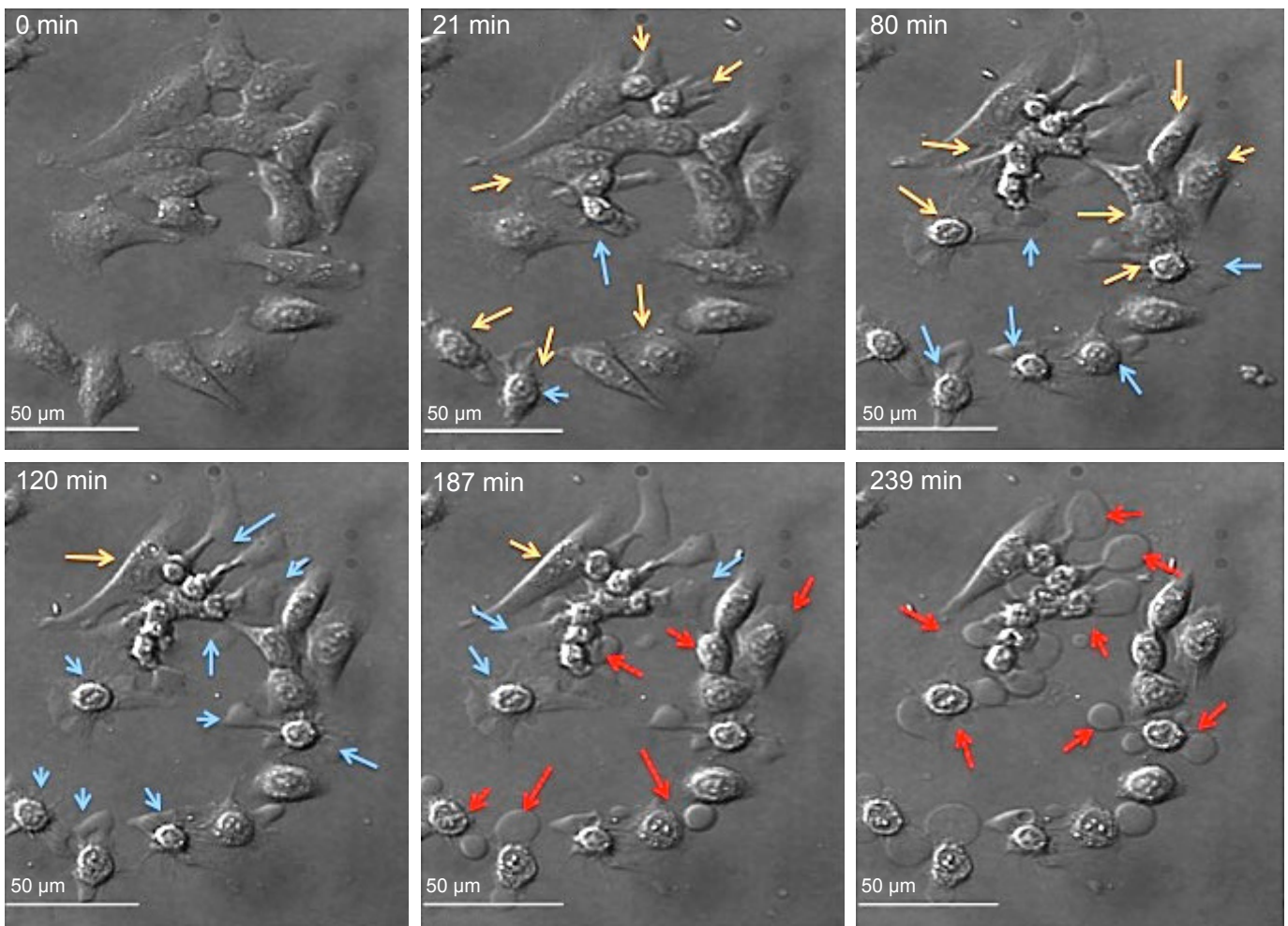

Figure 7 Time-lapse images of HCT-II6 cells incubated with SiNP-PAG9 show loss of cell adhesion (yellow arrows), blebbing-like activity (blue arrows), and cell swelling (red arrows).

Abbreviations: SiNP, silica nanoparticle; PAG, photoacid generator. 


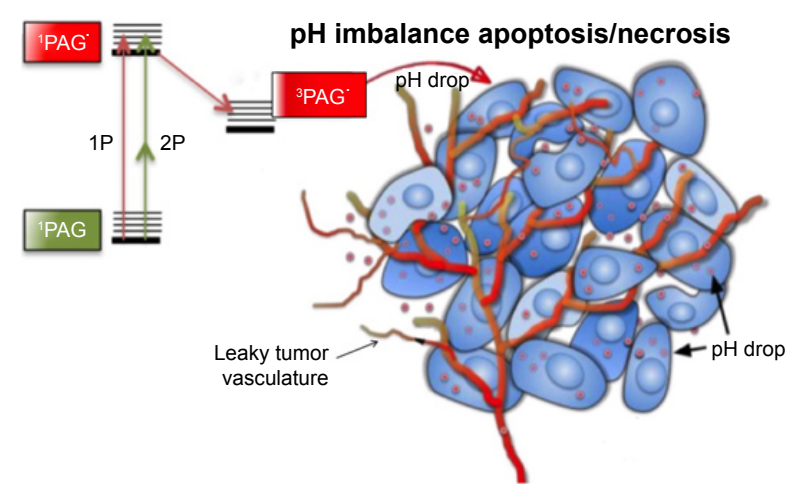

Figure 8 A schematic illustration (simplified Jablonski diagram) using one-photon (IP) or two-photon (2P) excitation PAG-based PDT to induce cell death by means of a $\mathrm{pH}$ imbalance.

Abbreviations: IP, one-photon; 2P, two-photon; PAG, photoacid generator; PDT, photodynamic therapy.

resulting in a higher cytotoxic effect. Ultimately, nearinfrared (IR) 2P excitation of the water-soluble PAG should afford deep tissue excitation and PDT. Thus, light-guided acid generation could be considered an attractive tool for treatment of hypoxic tumors and other diseases. Currently, our efforts are focused on evaluating the prepared PAGs in vivo and for $2 \mathrm{P}$ excitation in the near-IR spectral range.

\section{Acknowledgment}

We wish to acknowledge support from the National Science Foundation (CBET-1517273).

\section{Disclosure}

The authors report no conflicts of interest in this work.

\section{References}

1. Spikes JD. In: Berghausen RV, Jori G, Land EJ, Truscott TH, editors. Primary Photoprocesses in Biology and Medicine. New York, NY: Plenum Press; 1985:209-227.

2. Mroz P, Yaroslavsky A, Kharkwal GB, Hamblin MR. Cell death pathways in photodynamic therapy of cancer. Cancers. 2011;3(2):2516.

3. Zhu TC, Finlay JC, Hahn SM. Determination of the distribution of light, optical properties, drug concentration, and tissue oxygenation in-vivo in human prostate during motexafin lutetium-mediated photodynamic therapy. J Photochem Photobiol B. 2005;79(3):231.

4. Moore RB, Chapman JD, Mercer JR, et al. Measurement of PDT-induced hypoxia in Dunning prostate tumors by iodine-123-iodoazomycin arabinoside. J Nucl Med. 1993;34(3):405.

5. Brown JM, William WR. Exploiting tumour hypoxia in cancer treatment. Nat Rev Cancer. 2004;4(6):437.

6. Yue XL, Yanez CO, Yao S, Belfield KD. Selective cell death by photochemically induced pH imbalance in cancer cells. J Am Chem Soc. 2013; 135(6):2112.

7. Ferrari M. Cancer nanotechnology: opportunities and challenges. Nat Rev Cancer. 2005;5(3):161-171.

8. Ruoslahti E, Bhatia SN, Sailor MJ. Targeting of drugs and nanoparticles to tumors. J Cell Biol. 2010;188(6):759.

9. Yanez CO, Andrade CD, Belfield KD. Characterization of novel sulfonium photoacid generators and their microwave-assisted synthesis. Chem Comm. 2009;(7):827.

10. Tannock IF, Rotin D. Acid $\mathrm{pH}$ in tumors and its potential for therapeutic exploitation. Perspect Cancer Res. 1989;49(16):4373.

11. Haggie PM, Verkman AS. Unimpaired lysosomal acidification in respiratory epithelial cells in cystic fibrosis. J Biol Chem. 2009; 284(12):7681.
International Journal of Nanomedicine

\section{Publish your work in this journal}

The International Journal of Nanomedicine is an international, peerreviewed journal focusing on the application of nanotechnology in diagnostics, therapeutics, and drug delivery systems throughout the biomedical field. This journal is indexed on PubMed Central,

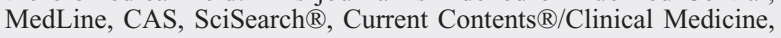

\section{Dovepress}

Journal Citation Reports/Science Edition, EMBase, Scopus and the Elsevier Bibliographic databases. The manuscript management system is completely online and includes a very quick and fair peer-review system, which is all easy to use. Visit http://www.dovepress.com/ testimonials.php to read real quotes from published authors. 